\title{
Subparsec-scale HI in the Nucleus of NGC 4151
}

\author{
C.G. Mundell ${ }^{1}$, J.M. Wrobel ${ }^{2}$, A. Pedlar ${ }^{3}$, J.F. Gallimore ${ }^{4}$ \\ ${ }^{1}$ ARI, Liverpool John Moores University, U.K. (cgm@astro.livjm.ac.uk) \\ ${ }^{2}$ NRAO, P.O. Box O, Socorro, NM 87801 \\ ${ }^{3} J B O$, Macclesfield, Cheshire, U.K. \\ ${ }^{4}$ NRAO, 520 Edgemont Rd, Charlottesville, VA 22903
}

\begin{abstract}
We present sensitive, high-resolution $\lambda 21-\mathrm{cm}$ VLBA+VLA observations of the radio jet and nuclear HI absorption in NGC 4151. The 25-mas (1.6-pc) resolution continuum image reveals a highly collimated radio jet, underlying the discrete components seen previously with MERLIN and the VLA. Spatially and kinematically complex HI absorption is detected against the whole 3-pc extent of the continuum component predicted by Ulvestad et al. to contain the AGN. Instead, we suggest the component against which the absorption is detected is part of the eastern counterjet, ruling it out as the location for the AGN.
\end{abstract}

\section{Introduction}

$\lambda 21-\mathrm{cm}$ MERLIN observations of the Seyfert galaxy NGC 4151 revealed localized and marginally resolved $\mathrm{HI}$ absorption, with a peak column density of $N_{\mathrm{H}} \sim 6 \times 10^{19} T_{\mathrm{S}} \mathrm{cm}^{-2}$, against the component $(\mathrm{C} 4)$ in the radio jet which is thought to contain the AGN (Mundell et al. 1995); no absorption was detected against the other jet components (Fig. 1a). An east-west column density gradient was observed and, in combination with UV column densities and early VLBI images (Fig. 1b), which showed C4 to consist of two components (C4E and $\mathrm{C} 4 \mathrm{~W}$ ), led Mundell et al. (1995) to suggest that the weaker, western component, C4W, contains the optical/UV nucleus and the HI absorption is taking place against the first component of the counterjet $(\mathrm{C} 4 \mathrm{E})$, due to gas in the obscuring torus (Fig. 1c). Structural and spectral index information obtained from subsequent radio continuum VLBA observations of the jet at 1.6 and $5 \mathrm{GHz}$ led Ulvestad et al. (1998) to suggest a similar model but with the AGN located at the emission peak of $\mathrm{C} 4 \mathrm{E}$; this model predicted an absence of $\mathrm{HI}$ absorption against the AGN, with HI absorption occurring only against the start of the counterjet, i.e. the tail of emission extending eastwards of the peak in C4E.

\section{Preliminary Results}

As shown in Fig. 1(f), HI absorption is detected against the whole extent of $\mathrm{C} 4 \mathrm{E}$, thereby ruling it out as the location of the AGN. The absorption is spa- 


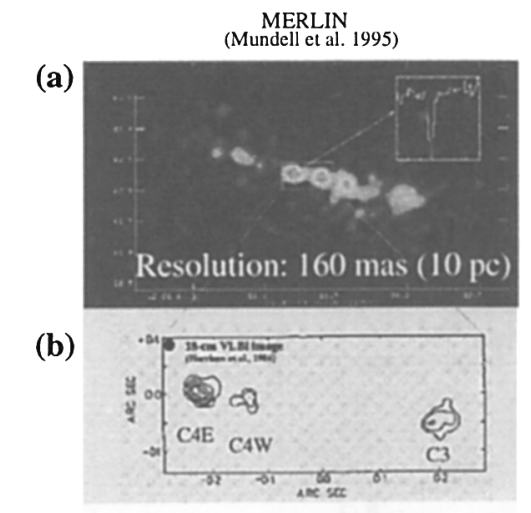

(c)

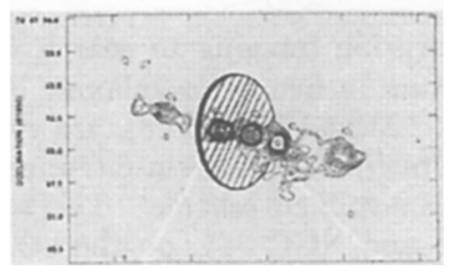

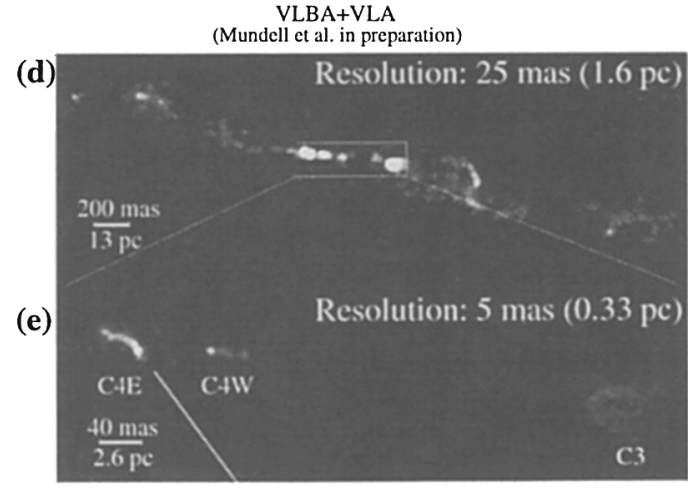

(f)

Figure 1. MERLIN: (a) $\lambda 21-\mathrm{cm}$ continuum image of radio jet with $\mathrm{HI}$ absorption against component $\mathrm{C} 4$ inset. (b) VLBI image of Harrison et al. with $\mathrm{C} 4$ resolved into $\mathrm{C} 4 \mathrm{E}$ and $\mathrm{C} 4 \mathrm{~W}$. (c) Model to explain $\mathrm{HI}$ absorption against first component in eastern counterjet (Mundell et al. 1995); VLA + VLBA: (d) New $\lambda 21-\mathrm{cm}$ continuum image of jet. (e) Full resolution image of components C4 and C3 showing same region as in (b). (f) HI absorption detected against full extent of $\mathrm{C} 4 \mathrm{E}$.

tially and kinematically complex with column densities $N_{\mathrm{H}} \sim 10^{20} T_{\mathrm{S}}$, where $T_{\mathrm{S}}$ is the spin temperature for which the value is unknown but is typically $\sim 10^{2}-10^{4}$ $\mathrm{K}$ in the Galaxy (Heiles \& Kulkarni, 1988). The VLBA+VLA continuum image also reveals a highly collimated radio jet, imaged over its full extent of $\sim 2^{\prime \prime} .5$ at milliarcsecond resolution (Fig. 1d,e), which, although less intrinsically luminous, seems to resemble those in quasars and radio galaxies; the brighter knots might correspond to shock features, coinciding with changes in direction, due to interaction with the ISM in the central $\sim 100 \mathrm{pc}$.

\section{References}

Harrison, B., Pedlar, A., Unger, S.W., Burgess, P., Graham, D.A. \& Preuss, E. 1986, MNRAS, 218, 775

Heiles, C. \& Kulkarni, S., 1988, in Galactic and Extragalactic Radio Astronomy, ed. K. Kellerman \& G. Verschuur, 95

Mundell, C.G., Pedlar A., Baum, S.A., O’Dea, C.P., Gallimore, J.F. \& Brinks, E. 1995, MNRAS, 272, 355

Ulvestad, J.S., Roy, A.R., Colbert, E.J.M. \& Wilson, A.S. 1998, ApJ, 496, 196 